\title{
The human skin microbiome
}

Allyson L. Byrd ${ }^{1-4}$, Yasmine Belkaid ${ }^{3,5}$ and Julia A. Segre

Abstract | Functioning as the exterior interface of the human body with the environment, skin acts as a physical barrier to prevent the invasion of foreign pathogens while providing a home to the commensal microbiota. The harsh physical landscape of skin, particularly the desiccated, nutrient-poor, acidic environment, also contributes to the adversity that pathogens face when colonizing human skin. Despite this, the skin is colonized by a diverse microbiota. In this Review, we describe amplicon and shotgun metagenomic DNA sequencing studies that have been used to assess the taxonomic diversity of microorganisms that are associated with skin from the kingdom to the strain level. We discuss recent insights into skin microbial communities, including their composition in health and disease, the dynamics between species and interactions with the immune system, with a focus on Propionibacterium acnes, Staphylococcus epidermidis and Staphylococcus aureus.

Our skin is home to millions of bacteria, fungi and viruses that compose the skin microbiota. Similar to those in our gut, skin microorganisms have essential roles in the protection against invading pathogens, the education of our immune system and the breakdown of natural products ${ }^{1-3}$. As the largest organ of the human body, skin is colonized by beneficial microorganisms and serves as a physical barrier to prevent the invasion of pathogens. In circumstances where the barrier is broken or when the balance between commensals and pathogens is disturbed, skin disease or even systemic disease can result. Human skin sites can be categorized by their physiological characteristics, that is, whether they are sebaceous (oily), moist or dry (BOX 1). Studying the composition of the microbiota at different sites is valuable for elucidating the aetiology of common skin disorders, which often have a preference for specific skin sites, such as eczema inside the elbow ${ }^{4}$ and psoriasis on the outside of the elbow ${ }^{5}$.

Traditionally, skin microbial communities were explored by use of culture-based methods. As this approach selects for microorganisms that thrive in artificial growth conditions, it underestimates the total diversity of the community. For example, the skin genus Staphylococcus is cultivated more easily than Propionibacterium spp. or Corynebacterium spp., which were frequently underestimated in culture-based surveys ${ }^{6}$. Thus, to circumvent the bias imposed by culture and to capture the complete diversity of the microbiome, investigators began applying sequencing methods. These original sequencing approaches utilized sequence variation in conserved taxonomic markers as molecular fingerprints to identify members of microbial communities $^{7}$. For bacteria, the $16 \mathrm{~S}$ ribosomal RNA (rRNA) gene is used, whereas for fungi, the internal transcribed spacer 1 (ITS1) region of the eukaryotic ribosomal gene is preferred ${ }^{8}$.

As sequencing technologies have advanced from Sanger sequencing to 454 pyrosequencing and then Illumina sequencing, this original approach has been regularly adapted to accommodate increasing read depths and shorter read lengths. This has been accomplished with new primers for shorter amplicons, clustering methods to overcome sequencing error and assembly methods to combine paired-end reads. With shorter amplicon lengths ( $~ 300$ bp compared with $>1,000$ bp in Sanger sequencing), only a subregion of the $16 \mathrm{~S}$ rRNA gene can be analysed. This requires optimized primers that bind to specific regions of the 16S rRNA gene to capture the genetic diversity of the bacterial population; primer pairs that are used in skin microbiome studies should be optimized to amplify and distinguish prevalent cutaneous microbial taxa $\mathrm{a}^{9-11}$. To date, the primary pipelines for analysing amplicon data are mothur ${ }^{12}$ and Qiime $^{13}$. Both methods use a read clustering approach by which clustered reads are compared with curated reference databases to classify communities at the genus and, when possible, the species level.

Most skin microbiome surveys have used amplicon sequencing. Over the past few years, however, major technical and analytical breakthroughs have enabled shotgun metagenomic sequencing studies. FIGURE 1 highlights the technical and procedural differences between amplicon and shotgun metagenomics and the different types of analyses that are possible with the data sets. As shotgun metagenomics does not sequence 
Microbiota

An aggregate of

microorganisms, including

bacteria, archaea, protists,

fungi and viruses.

Microbiome

The composition of all

microbial genes in a

community specific target regions, it simultaneously captures all genetic material in a sample, including human, bacterial, fungal, archaeal and viral, thus allowing relative kingdom abundances to be inferred, with the limitation that the DNA of different microorganisms may be differentially extracted depending on the sample preparation method ${ }^{14-16}$. Another advantage of shotgun metagenomic sequencing is that these data sets provide sufficient resolution to differentiate species and even strains within a species. This is crucial for identifying

\section{Box 1 | Skin physiology}

Structurally, the skin is composed of two distinct layers: the epidermis and dermis (Figure). The outermost layer (the epidermis) is composed of layers of differentiated keratinocytes. The top layer, or stratum corneum, is composed of terminally differentiated, enucleated keratinocytes (also known as squames) that are chemically crosslinked to fortify the barrier of the skin ${ }^{113}$.

In addition to this conserved layered structure, body sites provide diverse microenvironments that vary in ultraviolet light exposure, $\mathrm{pH}$, temperature, moisture, sebum content and topography ${ }^{22}$. On the basis of these characteristics, sites can be grouped into broad categories: sebaceous or oily (face, chest and back); moist (bend of elbow, back of knee and groin) and dry (volar forearm and palm). The environment of these sites is influenced by appendages, such as sweat glands, hair follicles and sebaceous glands. More abundant in moist sites, sweat glands are important for thermoregulation through the evaporation of water, which also acidifies the skin, making conditions unfavourable for the growth and colonization of certain microorganisms ${ }^{22}$. In addition, sweat contains antimicrobial molecules, such as free fatty acids and antimicrobial peptides, that inhibit microbial colonization ${ }^{114}$. Connected to the hair follicle and denser in oily sites, sebaceous glands secrete lipid-rich sebum, a hydrophobic coating that lubricates and provides an antibacterial shield to hair and skin.

Depending on the method used to sample the skin microbiota (swab, biopsy, surface scrape, cup scrub or tape strip), microorganisms residing at different depths or subcompartments of the skin are captured ${ }^{92,115-117}$. Although most major bacterial taxa are similarly identified regardless of sampling method ${ }^{92}$, some microorganisms are variably present at the surface compared with deeper skin layers ${ }^{118-120}$; this emphasizes the importance of maintaining consistent sampling techniques throughout a study. In general, the studies highlighted throughout this Review utilize methods that capture microorganisms on and within the stratum corneum; additional studies with more invasive sampling techniques are necessary to fully understand the spatial distribution of microorganisms in the skin.

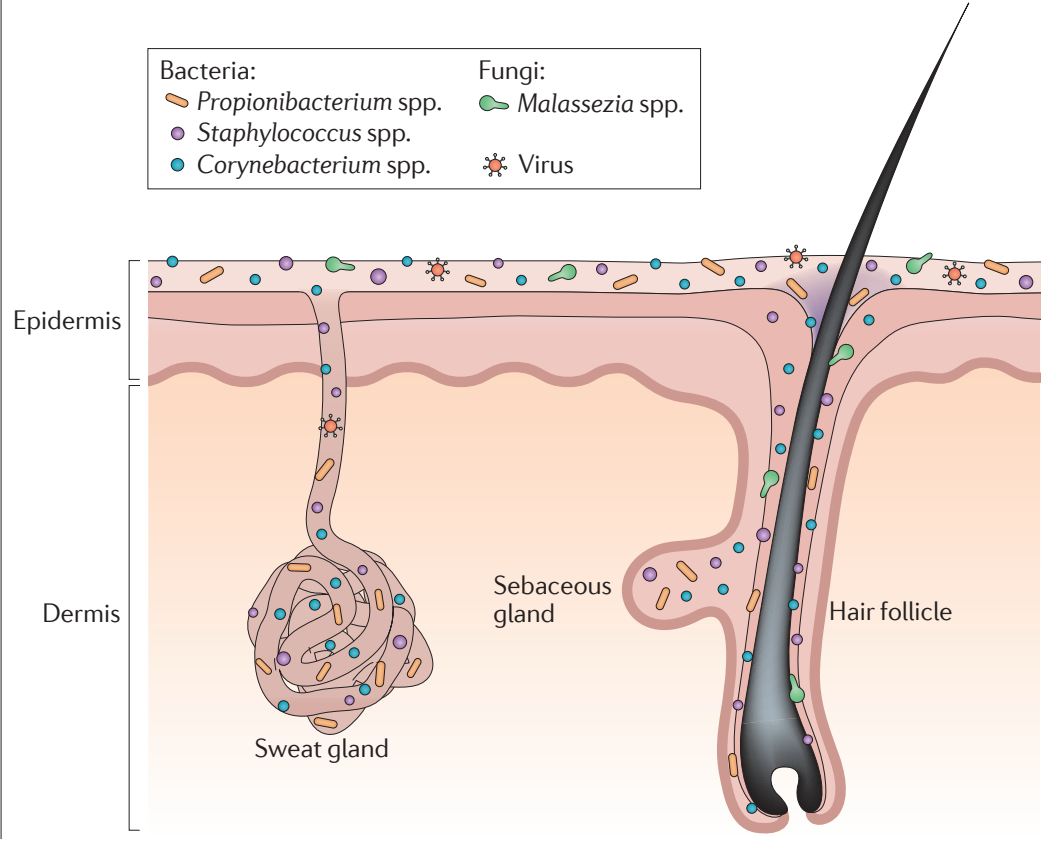

members of the Staphylococcus genus, which are difficult to classify to the species level with most amplicon sequencing approaches ${ }^{9}$. The ability to differentiate strains is important as more studies reveal the functional differences that exist between strains within a species ${ }^{17-19}$.

In this Review, we discuss recent insights into skin microbial communities, including their composition in health and disease, assembly and ecology, and interactions with the immune system. We end by considering important unanswered questions in the field and future research priorities. A greater understanding of these topics is important as interest in targeting the skin microbiome for therapeutic approaches increases.

\section{The skin microbiota in health}

Before investigating changes in the microbiota that are associated with a disease state, scientists must first establish a baseline and the normal variation in the microbiota of healthy individuals.

Composition of the skin microbiota. In sequencing surveys of healthy adults ${ }^{20-23}$, the composition of microbial communities was found to be primarily dependent on the physiology of the skin site, with changes in the relative abundance of bacterial taxa associated with moist, dry and sebaceous microenvironments. Sebaceous sites were dominated by lipophilic Propionibacterium species, whereas bacteria that thrive in humid environments, such as Staphylococcus and Corynebacterium species, were preferentially abundant in moist areas, including the bends of the elbows and the feet (FIG. 2; TABLE 1). In contrast to bacterial communities, fungal community composition was similar across core body sites regardless of physiology ${ }^{23,24}$. Fungi of the genus Malassezia predominated at core body and arm sites, whereas foot sites were colonized by a more diverse combination of Malassezia spp., Aspergillus spp., Cryptococcus spp., Rhodotorula spp., Epicoccum spp. and others $^{24}$ (FIG. 2). Bacteria were the most abundant kingdom across sites, and fungi were the least abundant ${ }^{25}$ (FIG. 2); however, there are many more bacterial reference genomes than fungal reference genomes available, which may partially contribute to this observed difference. Interestingly, the overall fungal abundance was low, even on the feet where fungal diversity was high.

In contrast to bacteria and fungi, colonization by eukaryotic DNA viruses was specific to the individual rather than anatomical site ${ }^{25}$ (FIG. 2). As no marker gene is universally shared among viruses, viral community diversity can be captured only with purified viral-like particles or shotgun metagenomics sequencing ${ }^{25,26}$. As an additional challenge, RNA viruses can be sequenced only with RNA sequencing, which has not been performed on skin samples from healthy individuals. Apart from bacteriophages, particularly those associated with Propionibacterium spp. and Staphylococcus spp., no core DNA virome has been found to be conserved across individual ${ }^{25,26}$. This area of skin microbiome research requires further attention to understand the role of possible predator-prey or cooperative interactions between bacteriophages and 


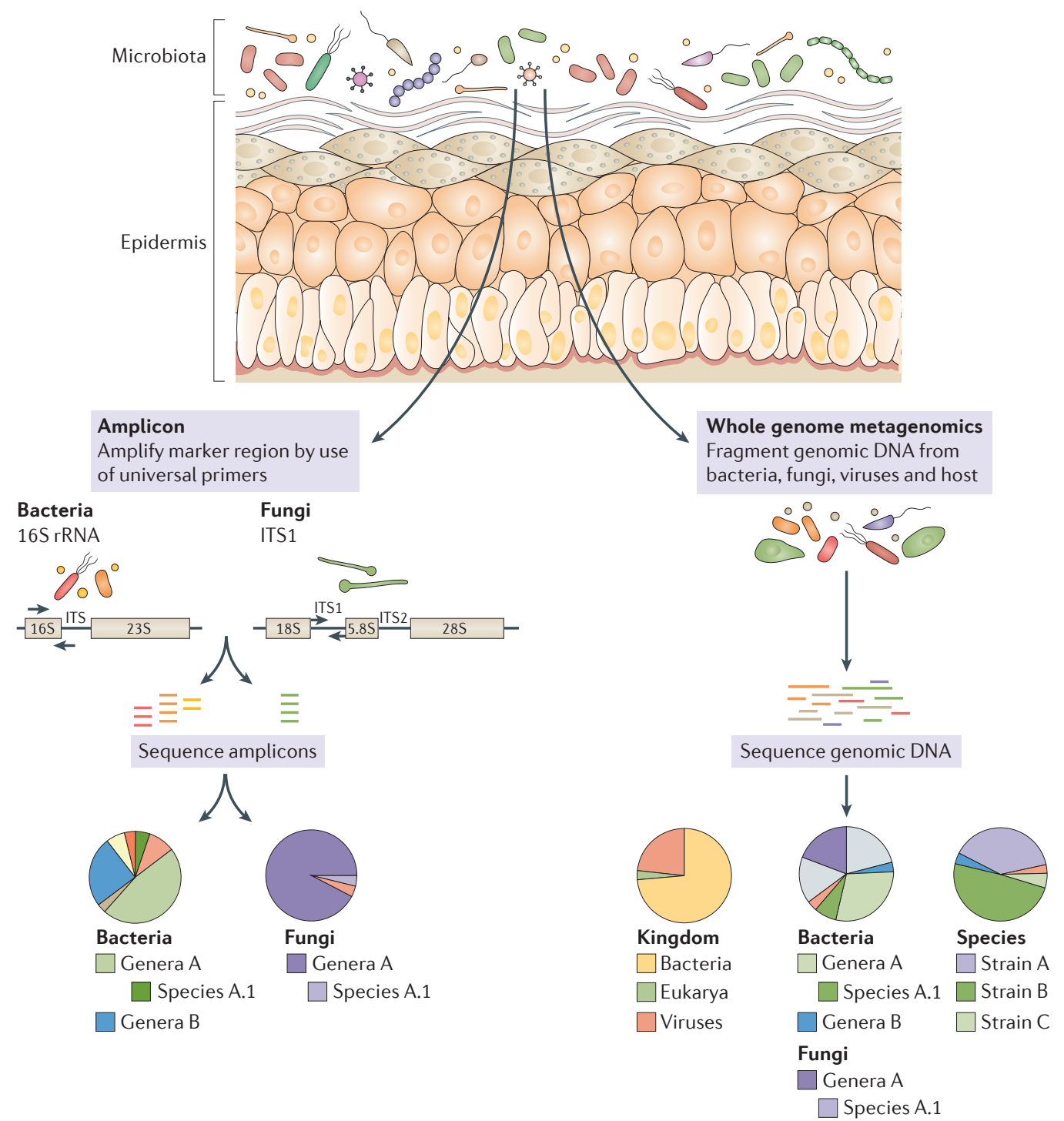

Amplicons

Segments of DNA or RNA that are targeted with primers and amplified in PCR.

Amplicon sequencing Querying microbial constituents of a community by targeted amplification and sequencing of a conserved marker gene.

\section{Reference genomes} Sequenced and assembled genomic content of a species with genes oriented as they appear on the chromosome.

Shotgun metagenomics sequencing

Unrestricted sequencing of all genomic material present in a clinical or an environmental sample.

\section{Virome}

The composition of all viral genes in a community.

Figure 1 | Amplicon versus shotgun metagenomic sequencing. To study the members of a microbial community, two sequencing strategies can be utilized. For amplicon sequencing (left), primers are utilized that amplify conserved regions within a kingdom. For bacteria, the $16 \mathrm{~S}$ ribosomal RNA (rRNA) region of the ribosomal gene is amplified, whereas for fungi, the internal transcribed spacer 1 (ITS1) region is amplified. By contrast, whole genome sequencing (right) captures the entire complement of genetic material in a sample without a targeted amplification step. Analyses of sequenced amplicons can identify the genus-level and the species-level community composition, but only shotgun metagenomics can reveal kingdom relative abundances and resolution to the strain level. Colours not defined may be grouped as 'Other'.

bacteria in microbial community assembly. In addition to bacteriophages, eukaryotic viruses may also have a role in skin diseases, as highlighted by the discovery of Merkel cell polyomavirus, an oncovirus that causes a rare but aggressive form of skin cancer ${ }^{27}$.

Through the use of longitudinal sampling, skin microbial communities were found to be largely stable over a 2-year study despite constant environmental changes $^{25}$. Based on analyses at the strain and singlenucleotide level, this stability was determined by the maintenance of strains over time rather than the reacquisition of common species from the environment ${ }^{25}$. Similarly, in longitudinal surveys of the gut, specific species of an individual's microbiota have been found to persist for a year ${ }^{28}$ or more ${ }^{29}$. Bacterial and fungal communities at sebaceous sites were the most stable. Microbial communities of foot sites were the least stable, and eukaryotic DNA viruses varied the most over time ${ }^{25,26}$. The relative instability of foot microbial communities might be attributable to the transient presence of fungi in the environment.

Beyond the species level, shotgun metagenomic data provide sufficient resolution to explore strain diversity of dominant skin bacterial species. Compared with the gut, skin has a low microbial biomass that leads to high host and low microbial sequencing depths 


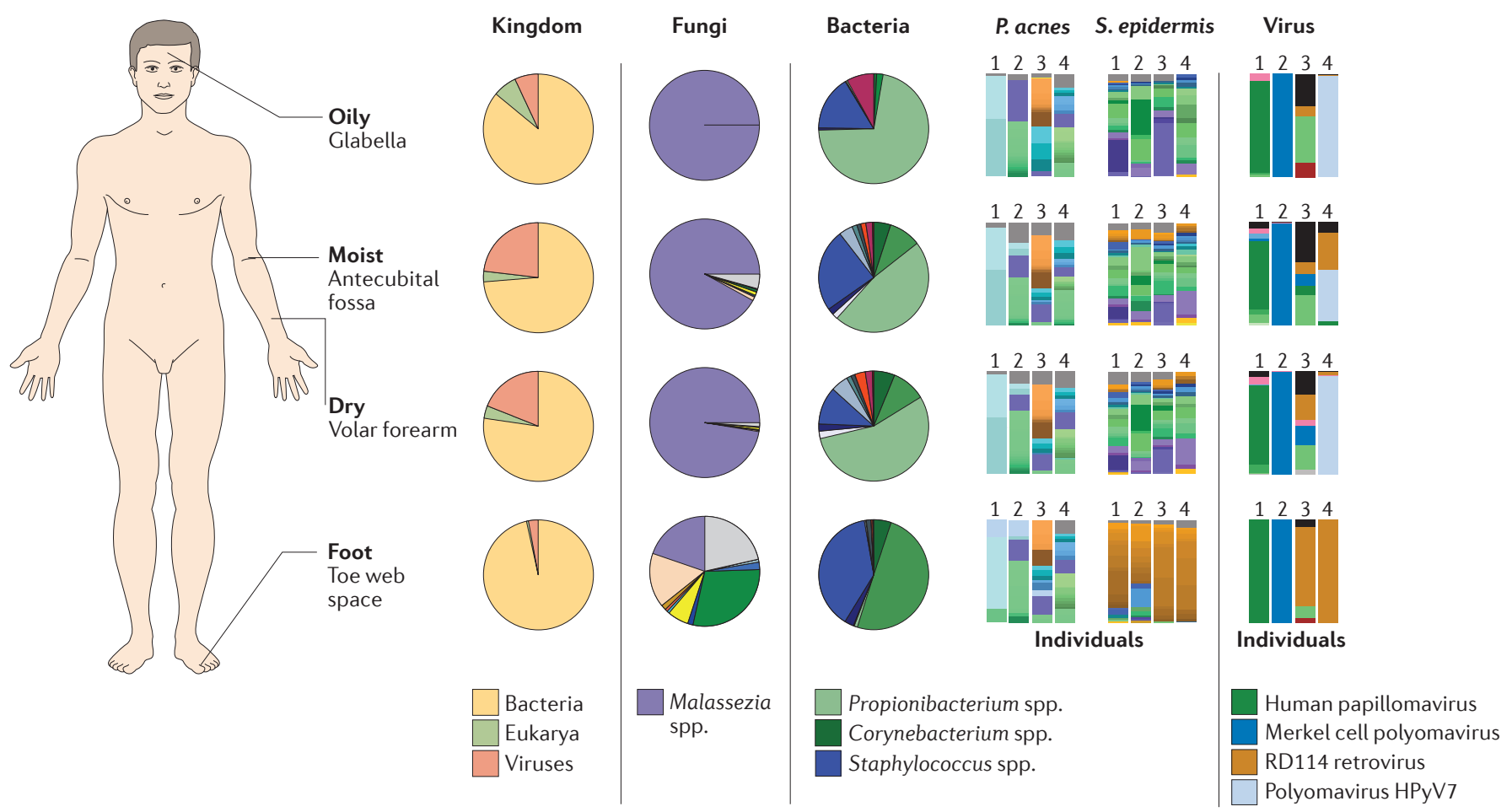

Colonization resistance A mechanism where commensal microorganisms prevent the colonization of harmful microorganisms.

\section{Prebiotic}

A substance, such as carbohydrate or fibre, that promotes the growth of beneficial bacteria.

\section{Probiotic}

Live microorganisms that are administered or consumed to confer a health benefit on the host.

Sebum

A mixture of lipids produced by sebaceous glands to lubricate and protect the skin.

\section{Stratum corneum}

The outermost layer of the epidermis composed of dead, mature skin cell keratinocytes.

Anoxic

A lack or absence of oxygen.

Sebaceous gland

An exocrine gland in the skin, usually attached to hair

follicles, that secretes sebum

Auxotrophic An organism that is unable to synthesize a particular compound required for its growth.

Figure 2 | Skin microbial communities are shaped by physiological characteristics and the individual. Four sites are shown to represent major microenvironments of the skin: glabella (also known as the forehead) sebaceous (oily); antecubital fossa (moist); volar forearm (dry); and toe web space (foot). Pie charts represent consensus relative abundances of the kingdom, fungi and bacteria across healthy adults ${ }^{2}$. The bacterial species Propionibacterium acnes and Staphylococcus epidermidis and eukaryotic DNA viruses are displayed as bar charts for four representative individuals to highlight how individuality shapes these communities ${ }^{25}$. For kingdom, fungi, bacteria and virus relative abundance plots, major taxa colours are identified in the legend. Unlabelled colours may be grouped as 'Other'. For the P. acnes and S. epidermidis bar charts, similar colours represent closely related strains.

in metagenomics samples. Despite low read depths, strain identification of prevalent skin species is possible because collections of sequenced reference genomes exist that span the diversity of a species ${ }^{17,18}$. Across body sites, individuals were found to be colonized by different multi-phyletic communities of Propionibacterium acnes and Staphylococcus epidermidis strains ${ }^{23}$ (FIG. 2). In addition, $S$. epidermidis strains from a commensal clade demonstrated site specificity with a tropism for human feet. Describing communities at strain-level resolution is important when the gene content differences between strains within a species can determine functional differences in health and disease. For example, antibiotic resistance and virulence genes are variably present in $P$. acnes and S. epidermidis strains ${ }^{17,18}$. Analysis of strain communities of other skin microbiota species will require the creation of additional comprehensive reference genome libraries or the development of new computational tools that are capable of classifying strains from low coverage metagenomic data. Currently, resolution at the strain level without mapping to reference genomes has been limited to single-molecule, long-read metagenomic sequencing studies ${ }^{30}$. Future functional studies will allow researchers to elucidate whether some strains provide colonization resistance against other strains of the same or different species as an important step in the development of prebiotic and probiotic strategies.
Compared with the richer environment of our intestines, skin lacks many nutrients beyond basic proteins and lipids. To survive in such a cool, acidic and desiccated environment (BOX 1), the resident microbiota of our skin have adapted to utilize the resources that are present in sweat, sebum and the stratum corneum ${ }^{1}$ (TABLE 1). For example, the facultative anaerobe $P$. acnes is able to thrive in the anoxic sebaceous gland by using proteases to liberate the amino acid arginine from skin protein $s^{31}$ and lipases to degrade triglyceride lipids in sebum $^{32}$; this releases free fatty acids, which promote bacterial adherence ${ }^{33-35}$. In facial samples, sebum levels of the cheek were shown to positively correlate with Propionibacterium spp. abundance ${ }^{36}$. Interestingly, for mammals such as mice, rats and dogs, which produce smaller quantities of triglyceride-rich sebum, $P$. acnes grows less well and is thus found at lower abundances ${ }^{37}$.

The lipids of sebum and the stratum corneum are also utilized by auxotrophic Malassezia and Corynebacterium species, as they are unable to produce their own lipids ${ }^{1}$. Corynebacterium spp. utilize these lipid compounds to generate the corynemycolic acids that coat their cell surface ${ }^{1}$. Consistent with the carbohydrate-deficient, lipid-rich environment of the skin, Malassezia spp. genomes are enriched for lipase genes and depleted for carbohydrate-utilizing enzyme genes compared with the genomes of other sequenced fungi $i^{38}$. Such differences 
Mycobiome

The composition of all fungal genes in a community. may explain why Malassezia species predominate in the adult skin mycobiome. Finally, Staphylococcus spp. have evolved many strategies for surviving on the skin, including the ability to be halotolerant (that is, to withstand the high salt content of sweat) and utilize the urea that is present in sweat as a nitrogen source ${ }^{1}$. To further promote colonization, various Staphylococcus spp. can also produce adherens that promote attachment to the skin and proteases that liberate nutrients from the stratum corneum ${ }^{1}$. Overall, the skin harbours a heterogeneous community of microorganisms that each have distinct adaptations to survive on the skin.

\section{Skin microbiota assembly and ecology}

Adults stably maintain the composition of their skin microbial communities as assessed for at least 2 years ${ }^{25}$; however, the forces that shape and maintain these complex communities remain poorly understood.

Initial colonization and population shifts. In newborn babies, initial colonization of the skin is dependent on delivery mode; neonates born vaginally acquire bacteria that colonize the vagina, whereas neonates born via Caesarian section acquire microorganisms that are associated with the $\operatorname{skin}^{39,40}$. The long-term effects of

Table 1 | Top ten abundant bacterial, eukaryotic and viral species that are present by physiological grouping of sites

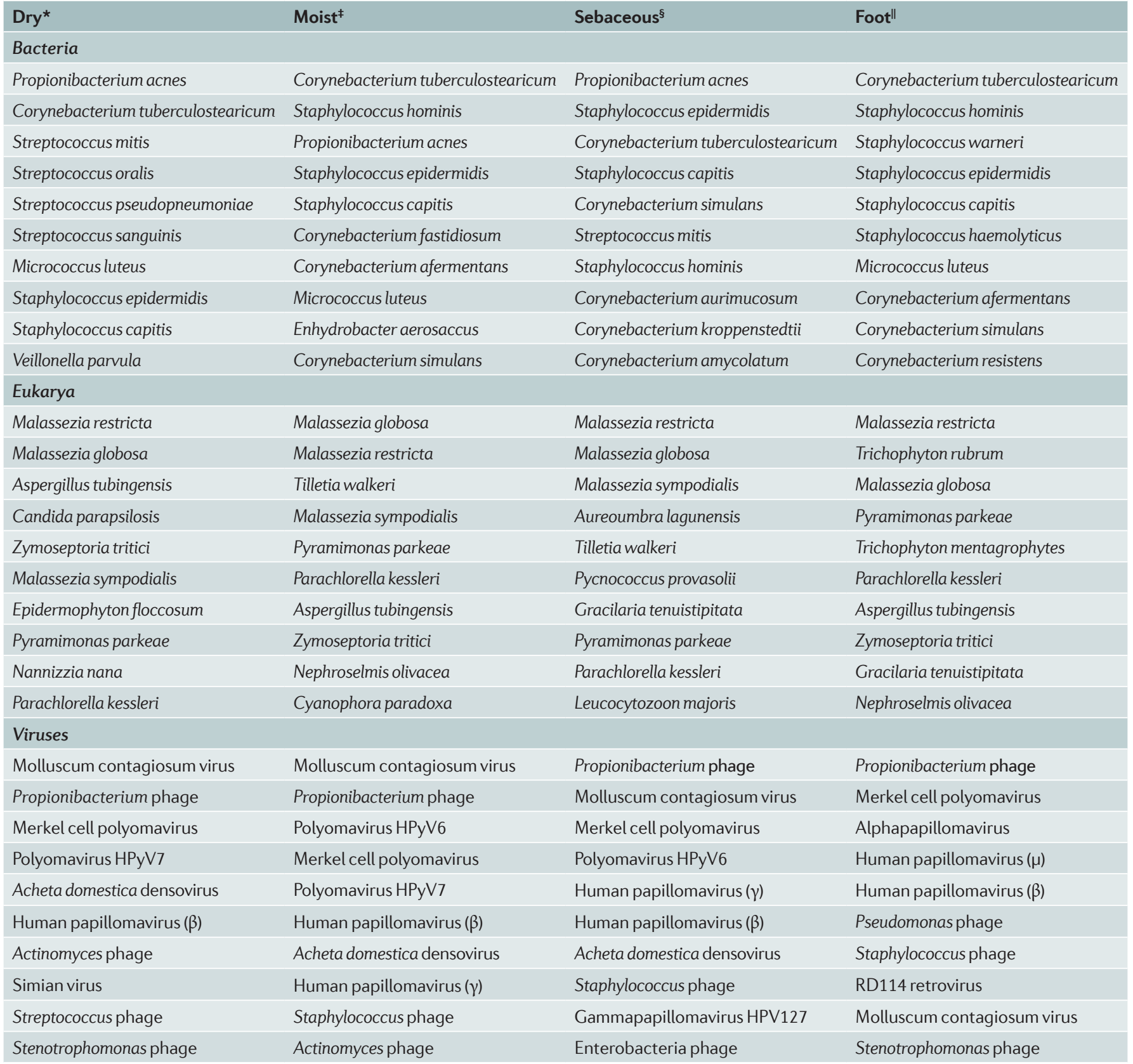

${ }^{\star}$ Hypothenar palm, volar forearm. ${ }^{\ddagger}$ Nare, antecubital fossa, inguinal crease, interdigital web, popliteal fossa. ${ }^{\S} \mathrm{Alar}$ crease, cheek, glabella, external auditory canal, manubrium, retroauricular crease, occiput, back. "Toe web space, toenail, plantar heel ${ }^{23}$. 
these initial skin colonization modes in neonates remain unknown, and shotgun metagenomic sequencing and strain-level analyses need to be carried out to address this outstanding issue.

In the gut, microbial communities stabilize around 3 years of age $e^{41}$. Before this stabilization, strains are likely acquired from close contacts and family members ${ }^{42}$ and are predicted to be maintained throughout life ${ }^{29}$. By contrast, the relative abundance of skin microbial species is restructured during puberty, a time when increased levels of hormones stimulate the sebaceous glands to produce additional sebum. Thus, the skin of postpubescent individuals favours the expansion of lipophilic microorganisms, such as bacterial Propionibacterium spp. and Corynebacterium spp. ${ }^{43}$ and fungal Malassezia spp. ${ }^{44,45}$. By contrast, pre-pubescent children have greater abundances of Firmicutes (Streptococcaceae spp.), Bacteroidetes and Proteobacteria (betaproteobacteria and gammaproteobacteria $)^{43}$ and a more diverse fungal community ${ }^{44}$. It remains unclear whether new strains are acquired during puberty or whether the relative abundance of existing strains changes. Overall, these agerelated changes in the skin microbiota are interesting, as many skin disorders are associated with age. For example, cases of Staphylococcus-associated atopic dermatitis decline in the majority of children before puberty, whereas Malassezia-associated tinea versicolor is more common in adults than children ${ }^{46-48}$. Further studies are also required to characterize the skin microbiota of elderly individuals as physiology and the predisposition to skin infections change.

Interactions between cutaneous microbial species. Microbial community assembly, stability and function are driven by host factors as well as the interactions between these microorganisms. Microorganisms can act competitively to exclude one another or synergistically for mutual benefits. In the skin, Staphylococcus aureus has been the focus of many colonization resistance studies. S. aureus colonizes the nares of one-third of the population, and its presence is a significant risk factor for subsequent infection ${ }^{49,50}$. In clinical infections, $80 \%$ of $S$. aureus bloodstream isolates match those identified in the nares of the individual ${ }^{49}$. Eradication of $S$. aureus in the nares of a surgical patient substantially reduces his or her susceptibility to invasive infections ${ }^{51}$.

As $S$. aureus frequently evolves resistance to antibiotics $^{52}$ and vaccine development has shown limited efficacy ${ }^{53,54}$, alternative eradication strategies, particularly those that use commensal microorganisms, are an active area of research ${ }^{55}$. These competition studies are similar to those that explored how soil microorganisms compete via antibiotic production ${ }^{56}$. The first microorganisms that were reported to inhibit $S$. aureus biofilm formation were a subset of $S$. epidermidis strains that express the serine protease glutamyl endopeptidase (Esp; also known as GluSE) ${ }^{57}$. It was later found that the S. epidermidis Esp of these strains degrades proteins that are crucial for $S$. aureus biofilm formation and host epithelial adhesion ${ }^{58}$; when the protease activity of Esp was combined with the antimicrobial activities of $\beta$-defensin, the resultant bactericidal activity was sufficient to kill S. aureus in biofilms (FIG. 3). Interestingly, the vast majority of sequenced $S$. epidermidis isolates contain the esp (gseA) gene ${ }^{17}$, but in a former study, only a subset expressed the gseA gene under the conditions tested $^{57}$. This discrepancy is an important reminder that the possession of a gene does not guarantee constitutive expression.

In a more recent study, Staphylococcus lugdunensis inhibited $S$. aureus growth through the production of the antibiotic lugdunin, a novel thiazolidine-containing cyclic peptide $^{59}$ (FIG. 3). Importantly for long-term therapeutic potential, after multiple generations, $S$. aureus never developed resistance to the antimicrobial effects of lugdunin or escaped the degradation induced by Esp. This lack of resistance is in sharp contrast to traditional antibiotics against which organisms evolve resistance and emphasizes that naturally derived products may be a more effective means to inhibit opportunistic pathogens.

In a separate study, multiple coagulase-negative Staphylococcus spp., S. epidermidis and S. hominis were shown to produce novel lantibiotics that were able to synergize with the human cathelicidin antimicrobial peptide LL-37 and to inhibit the growth of S. aureus ${ }^{60}$. Strains producing these lantibiotics were depleted in individuals with atopic dermatitis, who are frequently colonized with $S$. aureus. In addition, the topical application of these antimicrobial-producing strains decreased the colonization of $S$. aureus in a small number of individuals with atopic dermatitis, demonstrating the translational potential of a probiotic strategy ${ }^{60}$.

Notably, not all microorganisms inhibit S. aureus; rather, it was found that some Propionibacterium species could induce $S$. aureus aggregation and biofilm formation in a manner dependent on dose, growth phase and $\mathrm{pH}^{61}$ (FIG. 3). In a separate in vitro study, S. aureus was found to change from virulent to commensal when exposed to the commensal Corynebacterium striatum ${ }^{62}$. This ability to alter $S$. aureus behaviour opens the therapeutic option of modulating their behaviour rather than destroying the pathogen ${ }^{62}$.

Examples of interactions between other skin microorganisms also exist. Corynebacterium accolens modifies the local environment of the skin to inhibit growth of the opportunistic pathogen Streptococcus pneumo$n i a e^{63}$. This response was dependent on C. accolens using the lipase activity of LipS1 to release antibacterial free fatty acids from skin surface triacylglycerols. In another study, pairwise antagonism assays were performed with isolates from culture collections of $S$. epidermidis and $P$. acnes $^{64}$. One P. acnes clade I-2 exhibited selectively higher antimicrobial activity against $S$. epidermidis than other $P$. acnes clades, likely owing to a thiopeptide conserved across type I-2 strains. Conversely, the majority of $S$. epidermidis strains tested were capable of inhibiting $P$. acnes growth in vitro. The authors of this study computationally predicted a variety of different mobile genetic elements that could be responsible for this phenotype in different strains. In a broader study of 89 isolates from six Staphylococcus spp., $84 \%$ of the 


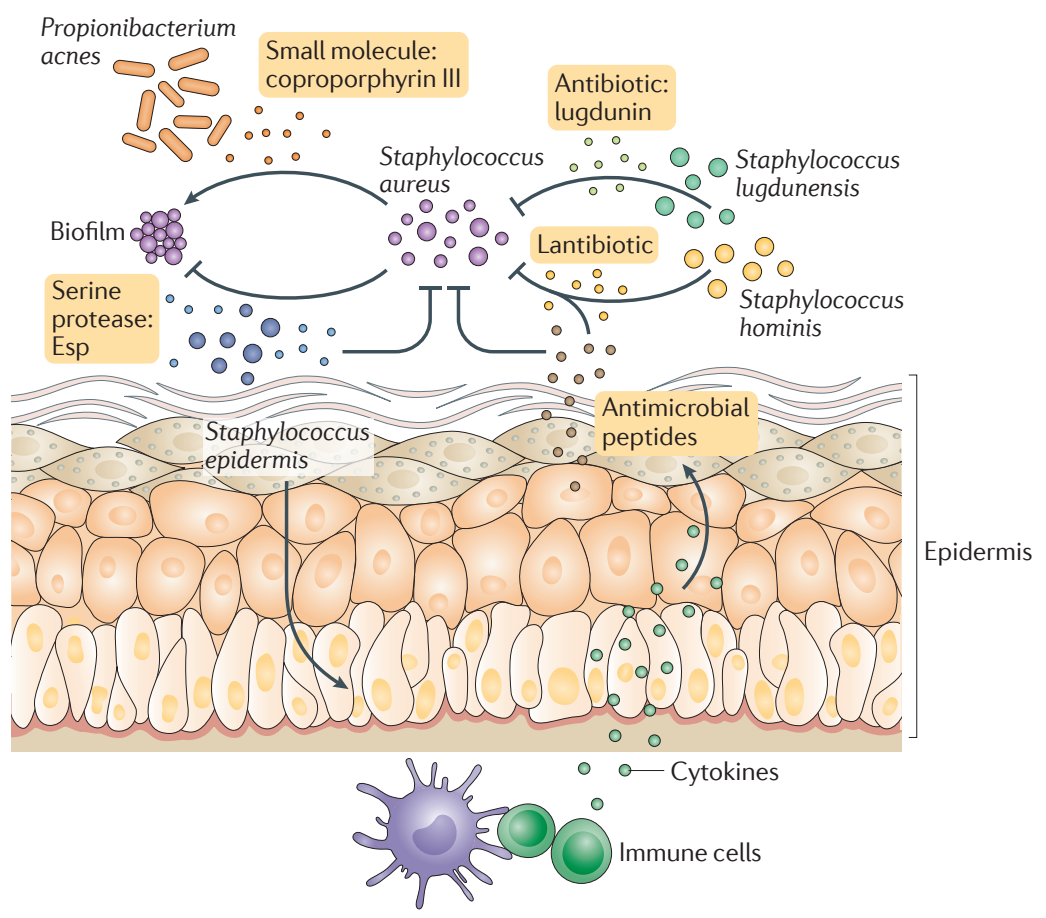

Figure 3 | Skin commensal interactions with Staphylococcus aureus. Skin microbial communities are shaped by interactions between organisms and with the host. In the skin, many interactions between commensals and Staphylococcus aureus have been identified. Antibiotics produced by coagulase-negative Staphylococcus and specifically by Staphylococcus lugdunensis prohibit colonization of S. aureus. Also, Staphylococcus epidermidis can inhibit S. aureus biofilm formation with production of the serine protease glutamyl endopeptidase (Esp). Moreover, when Esp-expressing S. epidermidis induces keratinocytes to produce antimicrobial peptides via immune cell signalling, S. aureus is effectively killed. In addition, Staphylococcus hominis-produced lantibiotics synergize with human antimicrobial peptide LL-37 to decrease S. aureus colonization. In contrast to inhibiting S. aureus, Propionibacterium acnes produces a small molecule, coproporphyrin III, that promotes S. aureus aggregation and biofilm formation.

\section{Bacteriocin}

An antimicrobial peptide produced by bacteria to inhibit or kill closely related or non-related bacteria.

\section{Dysbiosis}

A microbial community that is altered or impaired compared with normal. isolates could produce antimicrobial molecules that target common skin bacteria ${ }^{65}$, indicating that bacteriocin capacity is an evolutionary conserved trait among skin commensals.

In contrast to many inter-species interaction studies, investigations of the dynamics between strains within a species are rarer. Extrapolations from metagenomic data have revealed two patterns of strain colonization of the human infant gut. For some species, there is a single dominant strain, whereas for other species, multiple strains coexist at similar levels ${ }^{66}$.

In the skin, P. acnes and S. epidermidis exist as stable heterogeneous communities of strains ${ }^{23,25}$. Pan-genome analyses of $P$. acnes suggested that functional saturation, or containing the entire gene encoding potential found in the pan-genome of this species, drives the maintenance and acquisition of multiple bacterial strains ${ }^{25}$. Within the gut, studies have shown that a community of Clostridium species can act synergistically to enhance regulatory $\mathrm{T}$ cell responses to a greater extent than any individual species could alone ${ }^{67}$. In the skin, similar studies are needed to demonstrate the possible functional advantages of heterogeneous strain communities.

\section{The skin microbiome in disease}

Interactions between members of the microbiota both shape the resident microbial community and prevent colonization by pathogenic bacteria in a process termed 'colonization resistance' (REF. 68). However, in certain contexts, bacteria that are ordinarily beneficial to their hosts can become pathogenic. Many common skin diseases are associated with changes in the microbiota, termed dysbiosis ${ }^{69}$. This dysbiosis is often driven by common commensal species, as described below for acne, eczema and chronic wounds. Both rare and common skin disorders are thought to have underlying contributions both from individual species and from alterations to the microbial community. Additional longitudinal clinical studies may elucidate a mechanistic link between fungal species and dandruff or toenail infections and between viruses and warts.

Microorganisms associated with common acne. The prevalent teenage condition acne vulgaris is a chronic inflammatory skin condition that is associated with the bacterium P. acnes ${ }^{70}$, the most abundant organism in the microbiota of healthy adults ${ }^{18,71}$. At a functional level, gene expression profiles of $P$. acnes are distinct between individuals with acne and individuals without acne $\mathrm{e}^{72}$. The observation that almost all adults are colonized with $P$. acnes but only a minority have acne highlights the importance of studying diseases in the broader context of host genetics, immune or barrier defects, the microbiome and the environment. For example, increased sebum secretion is associated with the pathophysiology of acne, as secretion rates correlate with the severity of clinical symptoms ${ }^{73}$. In a study using fluorescent microscopy to visualize $P$. acnes in follicles of skin biopsy samples, acne development was substantially associated with the presence of $P$. acnes in follicles and its formation of biofilms ${ }^{74}$. At the clade level, $P$. acnes belonging to the type $1 \mathrm{~A}_{1}$ phylogroup have been consistently associated with acne across studies utilizing distinct sampling and analysis methods ${ }^{71,75-77}$. Strains within the type $1 A_{1}$ phylogroup have increased inflammatory potential based on the presence of putative virulence factors that affect bacterial adhesion and host immune responses ${ }^{78}$.

Historically, vitamin $B_{12}$ supplementation has been associated with acne in a subset of individuals ${ }^{79-83}$. Recently, this has been linked to supplemental vitamin $B_{12}$ repressing vitamin $B_{12}$ biosynthesis in P. acnes, which subsequently increases the production of porphyrins that can induce skin inflammation and acne development ${ }^{72}$. Interestingly, acne-associated $P$. acnes strains were found to produce substantially higher levels of porphyrins ${ }^{84}$.

Staphylococcus aureus and atopic dermatitis. Atopic dermatitis (also known as eczema) is a chronic, relapsing inflammatory disease with multiple contributing factors, including epidermal barrier impairment, immune cell activation and alterations in the community of associated skin microorganisms. Atopic dermatitis susceptibility has been associated with mutations 
in over 30 host gene loci, including the gene encoding skin barrier protein filaggrin ${ }^{85}$ and genes linked to the immune system ${ }^{86}$. In addition to $S$. aureus, which is commonly cultured from the skin of individuals with atopic dermatitis ${ }^{87}$, there are additional factors that support the hypothesis that microbiota have an influential role in disease pathogenesis. Atopic dermatitis is clinically treated with emollients that promote barrier integrity and immunosuppressive medications, such as steroids ${ }^{88}$. In cases where there is an infection or disease persistence, antimicrobial approaches (for example, antibiotics and dilute bleach baths) may be used, and their success has been shown to correlate with decreases in the relative abundance of S. aureus ${ }^{4}$; however, their overall effectiveness is uncertain ${ }^{89}$. As described above, much research is aimed to develop novel therapies specific to anti-S. aureus to replace the more broad-spectrum antimicrobials that are currently used.

In longitudinal studies of paediatric individuals with atopic dermatitis, 16S rRNA and whole genome sequencing of clinical samples showed that the relative abundance of Staphylococcus spp., particularly $S$. aureus and $S$. epidermidis, increased in the flare (episodic exacerbation) versus the post-flare state and that the relative abundance of staphylococci correlated with more severe disease at flare ${ }^{4,90}$. At the strain level, individuals with atopic dermatitis were found to be colonized with heterogeneous communities of S. epidermidis, and those with more severe disease were colonized with dominant $S$. aureus strains $s^{90}$. The correlation of $S$. aureus with atopic dermatitis during active disease exacerbation is well documented. However, the functional role of staphylococci in driving the atopic dermatitis disease state is poorly understood. Longitudinal sampling at more frequent intervals before a flare is still needed to identify whether increased staphylococci levels precede clinical symptoms, which would support the notion that staphylococci contribute to the initial onset of inflammation rather than bloom as a consequence of it. This warrants further investigation, as preliminary studies found a greater abundance of Staphylococcus spp. at 2 months in infants who did not develop atopic dermatitis by age 1 than in those who did develop atopic dermatitis by age 1 . This suggests that Staphylococcus spp. exposure at an early age is helpful for proper education of the immune system ${ }^{91}$.

Another genome sequencing study compared the unaffected skin of adults with atopic dermatitis with that of a control cohort and identified an enrichment of Streptococcus spp. and Gemella spp. and a depletion of Dermacoccus spp. in individuals prone to atopic dermatitis ${ }^{92}$. At a functional level, the study showed that the microbiome of these individuals is primed to generate excess ammonia, providing an explanation for the high $\mathrm{pH}$ levels that are observed during atopic dermatitis flares ${ }^{92}$.

The decreased diversity of the skin microbiome in individuals with atopic dermatitis has been linked to a reduction in environmental biodiversity in the areas surrounding their homes ${ }^{93}$. In one study, healthy individuals had greater diversity of gammaproteobacteria in their skin, the presence of which correlated with greater IL-10 expression in blood ${ }^{93}$. A follow-up study using in vitro and in vivo animal experiments showed that the gammaproteobacteria genus Acinetobacter could induce strong $\mathrm{T}$ helper $1\left(\mathrm{~T}_{\mathrm{H}} 1\right)$ and anti-inflammatory immune responses that were protective against allergic inflammation ${ }^{94}$. In a study that examined the microbiome of unaffected skin of individuals with ichthyosis vulgaris and a filaggrin deficiency, there was an underrepresentation of Gram-positive anaerobic cocci compared with their presence in healthy controls, indicating that a defective stratum corneum is sufficient to alter the skin microbiome and may drive the dysbiosis that is associated with eczema ${ }^{95}$.

Owing to the association of $S$. aureus with atopic dermatitis, other skin diseases and bloodstream infections, many studies have focused on interactions between $S$. aureus, its toxins and the immune system. For example, $S$. aureus $\delta$-toxin induces the degranulation of mast cells, which promotes both innate and adaptive type two immune responses ${ }^{96}$. S. aureus a-toxin can also induce IL- $1 \beta$ production from monocytes, which may consequently promote a $\mathrm{T}_{\mathrm{H}} 17$ response, or from $\mathrm{CD}^{+} \mathrm{T}$ cells making the cytokine IL-17 (REF. 97). By contrast, when exposed to $S$. aureus-derived cell wall component lipoteichoic acid, T cells neither proliferated nor produced cytokines ${ }^{98}$, indicating that $S$. aureus products can activate the immune system and also temporarily paralyse it. In addition to targeting immune cells, $S$. aureus has also been shown to trigger adipocytes to rapidly proliferate and to produce increased levels of the antimicrobial peptide cathelicidin as a host defence mechanism ${ }^{99}$. These examples demonstrate the many ways that $S$. aureus could initiate or exacerbate skin disorders in the broader context of barrier defects or altered immunity. In fact, it has been demonstrated that in the context of barrier defects, $S$. aureus is able to traverse the epidermis into the dermis, where it encounters immune cells and triggers the expression of the inflammatory cytokines IL-4, IL-13 and IL-22 and thymic stromal lymphopoietin ${ }^{100}$. Notably, the ability of $S$. aureus to trigger the cutaneous immune response can be strain-dependent ${ }^{90}$, highlighting the importance of evaluating a phenotype across isolates of a species. Although many of these experiments were performed in murine models, they are relevant to humans, as many of the pathways underlying inflammation and immunity in murine skin appear relevant in human infection and disease. Additional examples of interactions between skin microorganisms and immune cells are discussed in BOX 2.

Although the inflammatory potential of S. aureus has been demonstrated and dysbiosis is common to many skin diseases, it is still unknown whether these changes are a consequence of the disease state or whether $S$. aureus contributes to the initiation of the disease. Experiments with mouse models that are genetically and physically challenged to produce skin barrier or immunological defects have been used to determine the contribution of the microbiota to skin disease. For 
example, mice deficient in disintegrin and metalloproteinase domain-containing protein 17 (ADAM17) developed eczematous dermatitis as a consequence of microbial dysbiosis ${ }^{101}$. Alterations in cutaneous microbial communities, characterized by an overgrowth of Corynebacterium mastitidis, Corynebacterium bovis and S. aureus, preceded the development of features of atopic dermatitis. Targeted antibiotic treatment of these animals was sufficient to reverse the dysbiosis and eliminate skin inflammation, thus demonstrating a causal link between skin barrier alterations, dermatitis and the microbiome.

\section{Box $2 \mid$ Crosstalk between the immune system and the skin microbiota}

The immune system has evolved closely with resident microorganisms in the skin to allow the maintenance of commensal partners and the elimination of possible pathogens. To operate optimally, the skin microbiota, epithelial cells and innate and adaptive arms of the immune system need to communicate effectively. Keratinocytes can begin this dialogue by sensing microorganisms, especially pathogen-associated molecular patterns (PAMPs), through pattern recognition receptors (PRRs) ${ }^{22}$. Binding of PAMPs to PRRs triggers innate immune responses, resulting in the secretion of antimicrobial peptides that can rapidly kill and inactivate a diverse range of microorganisms, including fungi, bacteria and parasites. As a first line of defence against pathogens, some antimicrobial peptides are constitutively expressed ${ }^{114}$, whereas the expression of others can be transient and controlled by members of the skin microbiota ${ }^{121,122}$.

Cutaneous commensals are essential for education of the immune system ${ }^{123}$. During the postnatal period, the immature immune system allows microbial colonization in the absence of inflammatory responses ${ }^{124}$. This tolerance is dependent on regulatory T cells, a subset of lymphocytes that have been shown in mice to infiltrate neonatal skin, concomitant with hair follicle morphogenesis and skin microbial colonization ${ }^{125}$.

After this initial tolerogenic period, different microorganisms have been shown to elicit distinct effects on the immune system. For example, skin colonization with Staphylococcus epidermidis has been shown to induce increased levels of the cytokine interleukin-1 $\alpha(\mathrm{IL}-1 \alpha)^{122,123}$, which promotes skin-homing T cells to produce cytokines that contribute to host defence and skin inflammation ${ }^{122,123}$. Notably, under steady-state conditions, induction of effector $\mathrm{T}\left(\mathrm{T}_{\text {eff }}\right)$ cells in response to skin microorganisms occurs in the absence of classical inflammation in a process termed 'homeostatic immunity' (REFS 126,127) (Figure, left). This process represents an essential mechanism whereby different commensals educate distinct aspects of the immune system to respond to future pathogen exposures ${ }^{122,123}$. In other words, immune responses to pathogens occur in the context of broader recall responses to diverse microbial antigens ${ }^{128}$. This concept was demonstrated when mice pre-associated with bacterial S. epidermidis were better protected against fungal and parasitic skin infections ${ }^{122,123}$. By contrast, when S. epidermidis was introduced through intradermal injection to mice (instead of topically), classical inflammatory responses, as characterized by infiltrating monocytes and neutrophils, were observed alongside interferon- $\gamma$ (IFN $\gamma$ )-producing $T_{\text {eff }}$ cells (REF. 122) (Figure, right). Such contextual responses are essential so that S. epidermidis is maintained as a commensal on the skin surface but targeted by the immune system in the context of a barrier breach ${ }^{129}$.

Several studies demonstrate the distinct effects that microorganisms can have on the immune system. Now, future studies are needed to explore the microbial molecules that mediate these responses and how the immune system senses their presence. In addition, immunological tools should be developed to track these commensal-specific immune responses ${ }^{130}$. Such tools need to allow the visualization of both microorganisms and immune cells in the tissue and identify how their function and location can be affected by infection or inflammation. Further research integrating the exploration of skin barrier function to immunological and microbial triggers is necessary to transition from observations to therapeutics.

Homeostatic immunity

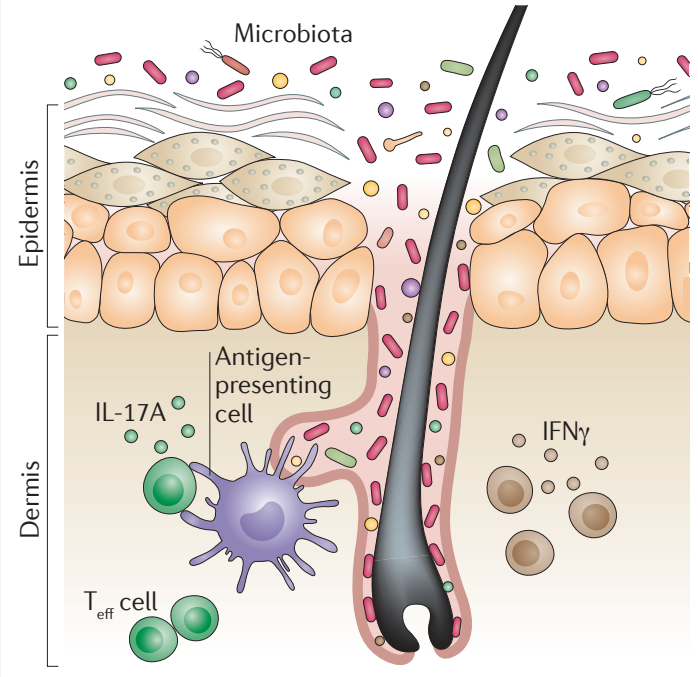

Inflammation

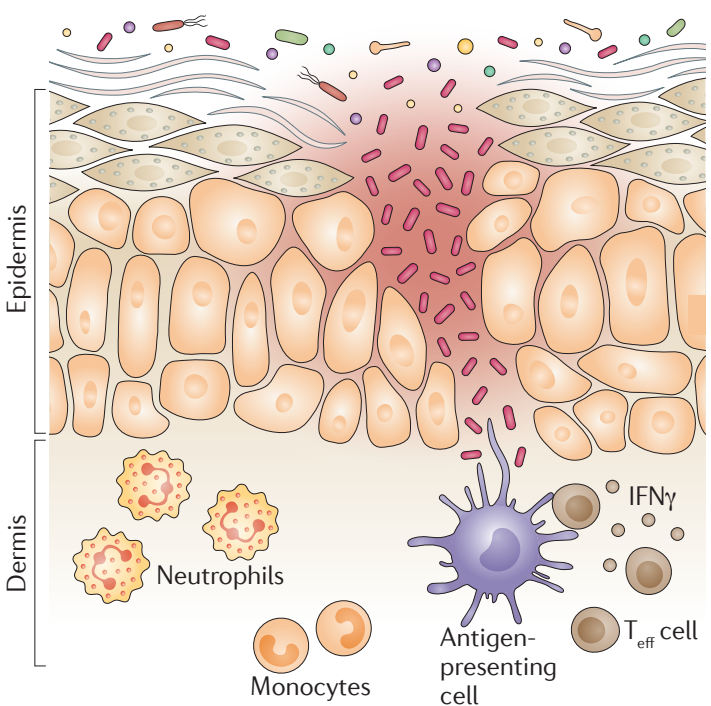




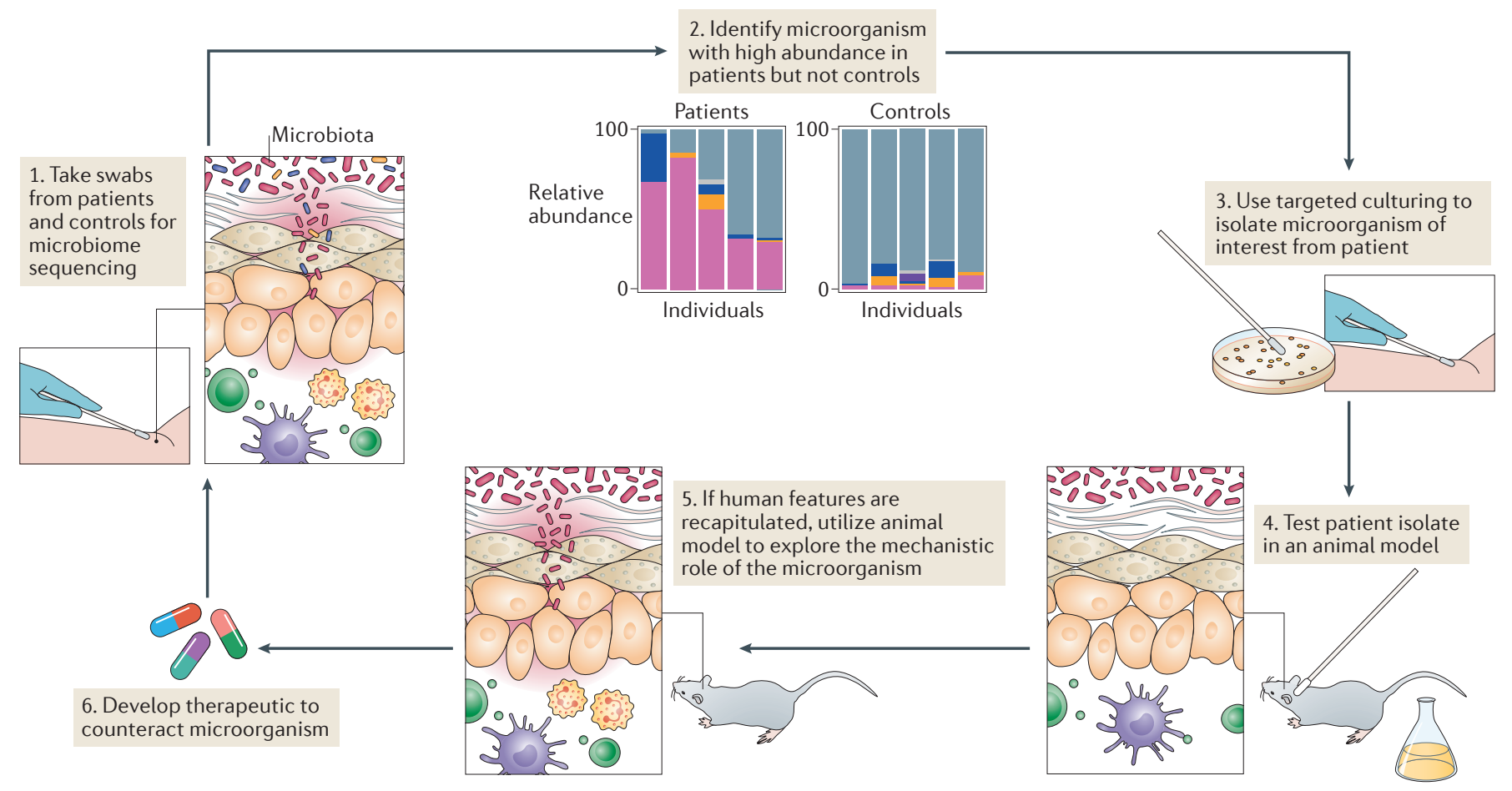

Figure 4 | Formulating testable hypotheses from sequencing data to generate novel therapeutics. To begin, skin swabs are taken from healthy controls and patients at sites relevant to the disease of interest (step 1). For example, the bend of the elbow and back of the knee would be relevant for atopic dermatitis. These skin samples are sequenced, and microbial communities between patients and controls are compared (step 2). Differences between groups can be used to generate hypotheses around microbial drivers of the disease. To experimentally test the hypotheses from computational analyses, isolates of interest should be cultured from patients (step 3). Targeted culture conditions can be used to more easily cultivate the isolate of interest. The patient-derived isolates should then be tested in preclinical models relevant to the disease (step 4). If the microorganism is sufficient to recapitulate features of the human disease, additional experiments should be utilized to explore the mechanism of action of the microorganism (step 5). On the basis of results from the previous steps, a therapeutic should be developed to counteract pathogenic microorganisms (step 6). A similar model could also be utilized to identify and evaluate protective, beneficial microorganisms present in controls but absent or under-represented in patients.

Skin microbiome of individuals with primary immunodeficiency. While several studies have investigated how microorganisms educate the immune system, the study of individuals with primary immunodeficiency (PID) provides an opportunity to understand the role of immunity in determining the structure of microbial communities. Underlying the rationale for these investigations are the common cutaneous manifestations of individuals with PID, particularly the eczematous features. To study this, skin microbiota samples were taken from individuals with rare monogenic PIDs, hyper immunoglobulin E (IgE) syndrome, Wiskott-Aldrich syndrome and dedicator of cytokinesis 8 syndrome. Despite distinct underlying mutations, all diseases are characterized by eczematous-like skin disease, reduced T and B cells, variable eosinophilia and elevated IgE levels ${ }^{102}$. Although overall similar in the types of bacteria that colonize the skin of healthy individuals, the skin of individuals with PID is more ecologically permissive with decreased temporal stability ${ }^{102}$. Despite individuals with PID being colonized with opportunistic fungi (for example, Candida spp. and Aspergillus spp.) and bacteria (for example, Serratia marcescens), which are typically absent in controls, these microorganisms still belong to phyla that are commonly associated with the skin. This suggests that organisms outside of these primary phyla are unable to stably survive in the nutrient-poor environments of the skin. In a separate study of individuals with PID caused by mutations in signal transducer and activator of transcription 1 (STAT1) or STAT3, the skin was colonized with more Gram-negative bacteria, particularly Acinetobacter spp., and there was a reduction in Corynebacterium spp. colonization compared with the levels in healthy controls ${ }^{103}$. To identify possible alterations in the viral communities, shotgun metagenomic sequencing of samples from these individuals is needed; these studies are clinically relevant, as some individuals with PID commonly suffer from viral skin infections ${ }^{104}$.

Microorganisms in chronic wound infections. In addition to classical skin diseases, microorganisms that colonize the skin have also been shown to affect the healing of chronic wounds prevalent in populations that are elderly or have diabetes or obesity. For example, the role of microorganisms has been well studied in the case of diabetic foot ulcers (DFUs). It is estimated that over $50 \%$ of DFUs are infected ${ }^{105}$. DFUs are a common result of diabetes-induced neuropathy and will 
occur in $15-25 \%$ of individuals with diabetes ${ }^{106}$, with $15.6 \%$ requiring amputation ${ }^{107}$. A $16 \mathrm{~S}$ rRNA sequencing survey found that bacterial communities colonizing neuropathic DFUs were associated with clinical features ${ }^{108}$. For example, shallow ulcers and those of short duration were associated with greater abundances of Staphylococcus spp., particularly S. aureus, whereas deeper ulcers and those of longer duration had greater microbial diversity and a higher relative abundance of anaerobic bacteria and Gram-negative Proteobacteria spp. ${ }^{108}$. In addition, poor control of blood glucose was associated with greater Staphylococcus spp. and Streptococcus spp. colonization ${ }^{108}$.

In a longitudinal survey of microorganisms associated with DFUs, $16 \mathrm{~S}$ rRNA sequencing of the wound revealed that bacterial community instability was associated with faster healing and more positive clinical outcomes ${ }^{109}$. This observation is counterintuitive, as many studies of other body sites have associated disease with bacterial community instability ${ }^{4,110}$. However, in the context of a wound, microbial instability could result in effective clearance of wound bacteria by the immune system. In addition to bacteria, the fungal community was also explored in the same cohort with amplicon sequencing of the ITS1 region ${ }^{111}$. Fungi were identified in $80 \%$ of the 100 DFUs analysed, with Cladosporium herbarum and Candida albicans identified as the most abundant species. In chronic wounds with poor clinical outcomes fungal diversity was increased and polymicrobial biofilms of fungi and bacteria were commonly found ${ }^{111}$.

\section{Conclusions and outlook}

In summary, this Review provides an analysis of the skin microbiome in health and disease at previously unexplored resolution. Analysis at this level was possible owing to technical advances in DNA extraction techniques and sequence library preparation methods that have been optimized for the diverse yet low biomass of skin samples. Moreover, the development of novel software pipelines that exploit the depth of information that is available in shotgun metagenomic sequencing data has advanced our understanding of the human skin microbiome. However, many questions remain regarding the function of the skin microbiota: what role do microorganisms have in the skin in maintaining health or promoting disease states?

DNA sequencing is a useful and unbiased tool for revealing the microorganisms in a sample; however, it is unable to differentiate between live colonizing and dead transient organisms. Although traditional culture techniques can distinguish between live and dead microorganisms, results are skewed by the culture conditions used. RNA sequencing may address this issue by revealing the functional activity of the microbiota, but it is technically difficult given the low biomass of organisms on skin. To measure this activity indirectly, a new analysis technique was developed that compares read distributions at the origin of replication and with those elsewhere in the genome as evidence of active bacterial replication ${ }^{112}$.

Analyses of microbiome sequencing data from patients compared with healthy controls can be used to generate hypotheses about putative disease-causing microorganisms (FIG. 4). Organisms of interest can then be isolated from individuals through targeted culturing methods. Next, these organisms can undergo whole genome sequencing to analyse their functional potential and can be tested in animal models to determine potential mechanistic roles in disease progression. Overall, the objective is to translate microbiome sequence data to functional studies that could inform the development of therapeutic modalities to ameliorate dysbiosis and counteract pathogens.
1. Scharschmidt, T. C. \& Fischbach, M. A. What lives on our skin: ecology, genomics and therapeutic opportunities of the skin microbiome. Drug Discov. Today Dis. Mech. 10, e83-e89 (2013).

2. Belkaid, Y. \& Segre, J. A. Dialogue between skin microbiota and immunity. Science 346, 954-959 (2014).

3. Grice, E. A. The intersection of microbiome and host at the skin interface: genomic- and metagenomic-based insights. Genome Res. 25, 1514-1520 (2015).

4. Kong, H. H. et al. Temporal shifts in the skin microbiome associated with disease flares and treatment in children with atopic dermatitis. Genome Res. 22, 850-859 (2012). This is the first study in which the skin of individuals with atopic dermatitis was longitudinally sampled and sequenced.

5. Paulino, L. C., Tseng, C. H., Strober, B. E. \& Blaser, M. J. Molecular analysis of fungal microbiota in samples from healthy human skin and psoriatic lesions. J. Clin. Microbiol. 44, 2933-2941 (2006).

6. Kong, H. H. \& Segre, J. A. Skin microbiome: looking back to move forward. J. Invest. Dermatol. 132, 933-939 (2012)

7. Woese, C. R. \& Fox, G. E. Phylogenetic structure of the prokaryotic domain: the primary kingdoms. Proc. Natl Acad. Sci. USA 74, 5088-5090 (1977). This is the first study that used the 16S rRNA gene to infer the phylogeny of a microorganism.

8. Schoch, C. L. et al. Nuclear ribosomal internal transcribed spacer (ITS) region as a universal DNA barcode marker for Fungi. Proc. Natl Acad. Sci. USA 109, 6241-6246 (2012).
9. Meisel, J. S. et al. Skin microbiome surveys are strongly influenced by experimental design J. Invest. Dermatol. 136, 947-956 (2016). This paper describes how skin microbiome studies can be affected by methodology.

10. Zeeuwen, P. L. et al. Reply to Meisel et al. J. Investigative Dermatol. 137, 961-962 (2017).

11. Castelino, M. et al. Optimisation of methods for bacterial skin microbiome investigation: primer selection and comparison of the 454 versus MiSeq platform. BMC Microbiol. 17, 23 (2017).

12. Schloss, P. D. et al. Introducing mothur: open-source, platform-independent, community-supported software for describing and comparing microbial communities. Appl. Environ. Microbiol. 75 , 7537-7541 (2009).

13. Caporaso, J. G. et al. QIIME allows analysis of high throughput community sequencing data. Nat. Methods 7, 335-336 (2010)

14. Brooks, J. P. et al. The truth about metagenomics: quantifying and counteracting bias in 16S rRNA studies. BMC Microbiol. 15, 66 (2015).

15. Yuan, S., Cohen, D. B., Ravel, J., Abdo, Z. \& Forney, L. J. Evaluation of methods for the extraction and purification of DNA from the human microbiome. PLOS ONE 7, e33865 (2012).

16. Gerasimidis, K. et al. The effect of DNA extraction methodology on gut microbiota research applications. BMC Res. Notes 9, 365 (2016).

17. Conlan, S. et al. Staphylococcus epidermidis pangenome sequence analysis reveals diversity of skin commensal and hospital infection-associated isolates. Genome Biol. 13, R64 (2012).
18. Tomida, S. et al. Pan-genome and comparative genome analyses of propionibacterium acnes reveal its genomic diversity in the healthy and diseased human skin microbiome. mBio 4, e000030-13 (2013).

19. Bosi, E. et al. Comparative genome-scale modelling of Staphylococcus aureus strains identifies strain-specific metabolic capabilities linked to pathogenicity. Proc. Natl Acad. Sci. USA 113, E3801-E3809 (2016).

20. Costello, E. K. et al. Bacterial community variation in human body habitats across space and time. Science 326, 1694-1697 (2009).

21. Grice, E. A. et al. Topographical and temporal diversity of the human skin microbiome. Science 324 1190-1192 (2009)

22. Grice, E. A. \& Segre, J. A. The skin microbiome Nat. Rev. Microbiol. 9, 244-253 (2011).

23. Oh, J. et al. Biogeography and individuality shape function in the human skin metagenome. Nature $\mathbf{5 1 4}$, 59-64 (2014).

24. Findley, K. et al. Topographic diversity of fungal and bacterial communities in human skin. Nature $\mathbf{4 9 8}$ 367-370 (2013)

25. Oh, J. et al. Temporal stability of the human skin microbiome. Cell 165, 854-866 (2016). Together with reference 23 , these studies explore the healthy human skin microbiome with shotgun metagenomic sequencing.

26. Hannigan, G. D. et al. The human skin doublestranded dna virome: topographical and temporal diversity, genetic enrichment, and dynamic associations with the host microbiome. $\mathrm{mBio} 6$ e01578-15 (2015). 
27. Feng, H., Shuda, M., Chang, Y. \& Moore, P. S. Clonal integration of a polyomavirus in human Merkel cell carcinoma. Science 319, 1096-1100 (2008)

28. Schloissnig, S. et al. Genomic variation landscape of the human gut microbiome. Nature 493, 45-50 (2013).

29. Faith, J. J. et al. The long-term stability of the human gut microbiota. Science 341, 1237439 (2013).

30. Tsai, Y. C. et al. Resolving the complexity of human skin metagenomes using single-molecule sequencing. $\mathrm{mBio}$ 7, e01948-15 (2016)

31. Holland, K. T., Greenman, J. \& Cunliffe, W. J. Growth of cutaneous propionibacteria on synthetic medium; growth yields and exoenzyme production. J. Appl. Bacteriol. 47, 383-394 (1979).

32. Bruggemann, H. et al. The complete genome sequence of Propionibacterium acnes, a commensal of human skin. Science 305, 671-673 (2004).

33. Marples, R. R., Downing, D. T. \& Kligman, A. M Control of free fatty acids in human surface lipids by Corynebacterium acnes. J. Invest. Dermatol. 56, 127-131 (1971)

34. Ingham, E., Holland, K. T., Gowland, G. \& Cunliffe, W. J. Partial purification and characterization of lipase (EC 3.1.1.3) from Propionibacterium acnes. J. General Microbiol. 124, 393-401 (1981).

35. Gribbon, E. M., Cunliffe, W. J. \& Holland, K. T Interaction of Propionibacterium acnes with skin lipids in vitro. J. General Microbiol. 139, 1745-1751 (1993).

36. Mukherjee, S. et al. Sebum and hydration levels in specific regions of human face significantly predict the nature and diversity of facial skin microbiome. Sci. Rep. 6, 36062 (2016)

37. Webster, G. F., Ruggieri, M. R. \& McGinley, K. J. Correlation of Propionibacterium acnes populations with the presence of triglycerides on nonhuman skin. Appl. Environ. Microbiol. 41, 1269-1270 (1981).

38. Wu, G. et al. Genus-wide comparative genomics of Malassezia delineates its phylogeny, physiology, and niche adaptation on human skin. PLoS Genet. 11, e1005614 (2015).

39. Dominguez-Bello, M. G. et al. Delivery mode shapes the acquisition and structure of the initial microbiota across multiple body habitats in newborns. Proc. Natl Acad. Sci. USA 107, 11971-11975 (2010).

40. Mueller, N. T., Bakacs, E., Combellick, J., Grigoryan, Z. \& Dominguez-Bello, M. G. The infant microbiome development: mom matters. Trends Mol. Med. 21 109-117 (2015)

41. Yatsunenko, T. et al. Human gut microbiome viewed across age and geography. Nature 486, 222-227 (2012).

42. Faith, J. J., Colombel, J. F. \& Gordon, J. I. Identifying strains that contribute to complex diseases through the study of microbial inheritance. Proc. Natl Acad. Sci. USA 112, 633-640 (2015).

43. Oh, J., Conlan, S., Polley, E. C., Segre, J. A. \& Kong, H. H. Shifts in human skin and nares microbiota of healthy children and adults. Genome Med. 4, 77 (2012).

44. Jo, J. H. et al. Diverse human skin fungal communities in children converge in adulthood. J. Invest. Dermatol. 136, 2356-2363 (2016)

45. Jo, J. H., Kennedy, E. A. \& Kong, H. H. Topographical and physiological differences of the skin mycobiome in health and disease. Virulence 8, 324-333 (2016)

46. Havlickova, B., Czaika, V. A. \& Friedrich, M Epidemiological trends in skin mycoses worldwide. Mycoses 51 (Suppl. 4), 2-15 (2008).

47. Seebacher, C., Bouchara, J. P. \& Mignon, B. Updates on the epidemiology of dermatophyte infections. Mycopathologia 166, 335-352 (2008)

48. Kyriakis, K. P. et al. Pityriasis versicolor prevalence by age and gender. Mycoses 49, 517-518 (2006).

49. von Eiff, C., Becker, K., Machka, K., Stammer, H. \& Peters, G. Nasal carriage as a source of Staphylococcus aureus bacteremia. N. Engl. J. Med. 344, 11-16 (2001).

50. Weidenmaier, C., Goerke, C. \& Wolz, C. Staphylococcus aureus determinants for nasal colonization. Trends Microbiol. 20, 243-250 (2012).

51. Bode, L. G. et al. Preventing surgical-site infections in nasal carriers of Staphylococcus aureus. N. Engl. J. Med. 362, 9-17 (2010).

52. DeLeo, F. R., Otto, M., Kreiswirth, B. N. \& Chambers, H. F. Community-associated meticillinresistant Staphylococcus aureus. Lancet 375 1557-1568 (2010).

53. Proctor, R. A Challenges for a universal Staphylococcus aureus vaccine. Clin. Infect. Dis. 54, 1179-1186 (2012).
54. Jansen, K. U., Girgenti, D. Q., Scully, I. L. \& Anderson, A. S. Vaccine review: "Staphyloccocus aureus vaccines: problems and prospects". Vaccine 31 2723-2730 (2013)

55. Pamer, E. G. Resurrecting the intestinal microbiota to combat antibiotic-resistant pathogens. Science 352 535-538 (2016)

56. Ling, L. L. et al. A new antibiotic kills pathogens without detectable resistance. Nature 517, 455-459 (2015)

57. Iwase, T. et al. Staphylococcus epidermidis Esp inhibits Staphylococcus aureus biofilm formation and nasal colonization. Nature 465, 346-349 (2010)

58. Sugimoto, S. et al. Staphylococcus epidermidis Esp degrades specific proteins associated with Staphylococcus aureus biofilm formation and hostpathogen interaction. J. Bacteriol. 195, 1645-1655 (2013).

59. Zipperer, A. et al. Human commensals producing a novel antibiotic impair pathogen colonization. Nature 535, 511-516 (2016)

Together with reference 57, these studies reveal how commensal skin microorganisms produce antimicrobials against $S$. aureus.

60. Nakatsuji, T. et al. Antimicrobials from human skin commensal bacteria protect against Staphylococcus aureus and are deficient in atopic dermatitis. Sci. Transl Med. 9, eaah4680 (2017). This is the first study that used autologous transplant of microorganisms to the skin.

61. Wollenberg, M. S. et al. Propionibacterium-produced coproporphyrin III induces Staphylococcus aureus aggregation and biofilm formation. $m$ Bio 5 , e01286-14 (2014).

62. Ramsey, M. M., Freire, M. O., Gabrilska, R. A., Rumbaugh, K. P. \& Lemon, K. P. Staphylococcus aureus shifts toward commensalism in response to Corynebacterium species. Front. Microbiol. 7, 1230 (2016).

63. Bomar, L., Brugger, S. D. Yost, B. H., Davies, S. S. \& Lemon, K. P. Corynebacterium accolens releases antipneumococcal free fatty acids from human nostril and skin surface triacylglycerols. mBio 7, e01725-15 (2016).

64. Christensen, G. J. et al. Antagonism between Staphylococcus epidermidis and Propionibacterium acnes and its genomic basis. BMC Genom. 17, 152 (2016).

65. Janek, D., Zipperer, A., Kulik, A., Krismer, B. \& Peschel, A. High frequency and diversity of antimicrobial activities produced by nasal Staphylococcus strains against bacterial competitors. PLoS Pathog. 12, e 1005812 (2016).

66. Yassour, M. et al. Natural history of the infant gut microbiome and impact of antibiotic treatment on bacterial strain diversity and stability. Sci. Transl Med. 8, 343 ra81 (2016).

67. Atarashi, K. et al. Treg induction by a rationally selected mixture of Clostridia strains from the human microbiota Nature 500, 232-236 (2013).

68. Buffie, C. G. \& Pamer, E. G. Microbiota-mediated colonization resistance against intestinal pathogens. Nat. Rev. Immunol. 13, 790-801 (2013).

69. lebba, V. et al. Eubiosis and dysbiosis: the two sides of the microbiota. New Microbiol 39, 1-12 (2016).

70. Leyden, J. J., McGinley, K. J., Mills, O. H. \& Kligman, A. M. Propionibacterium levels in patients with and without acne vulgaris. J. Invest. Dermatol. 65, 382-384 (1975).

71. Fitz-Gibbon, S. et al. Propionibacterium acnes strain populations in the human skin microbiome associated with acne. J. Invest. Dermatol. 133, 2152-2160 (2013).

72. Kang, D. Shi, B., Erfe, M. C., Craft, N. \& Li, H. Vitamin $B_{12}$ modulates the transcriptome of the skin microbiota in acne pathogenesis. Sci. Transl Med. 7, 293ra 103 (2015)

This is the first skin RNA sequencing study to compare microbial gene expression in individuals with and without acne.

73. Picardo, M., Ottaviani, M., Camera, E. $\delta$ Mastrofrancesco, A. Sebaceous gland lipids. Dermatoendocrinol. 1, 68-71 (2009).

74. Jahns, A. C. et al. An increased incidence of Propionibacterium acnes biofilms in acne vulgaris: a case-control study. Br. J. Dermatol. 167, 50-58 (2012).

75. Lomholt, H. B. \& Kilian, M. Population genetic analysis of Propionibacterium acnes identifies a subpopulation and epidemic clones associated with acne. PLOS ONE 5, e12277 (2010)
76. McDowell, A et al. An expanded multilocus sequence typing scheme for Propionibacterium acnes: investigation of 'pathogenic', 'commensal' and antibiotic resistant strains. PLOS ONE 7, e41480 (2012).

77. McDowell, A., Nagy, I., Magyari, M., Barnard, E. $£$ Patrick, S. The opportunistic pathogen Propionibacterium acnes: insights into typing, human disease, clonal diversification and CAMP factor evolution. PLoS ONE 8, e70897 (2013).

78. Omer, H., McDowell, A. \& Alexeyev, O. A Understanding the role of Propionibacterium acnes in acne vulgaris: The critical importance of skin sampling methodologies. Clin. Dermatol. 35, 118-129 (2017).

79. Balta, I. \& Ozuguz, P. Vitamin $B_{12}$-induced acneiform eruption. Cutane. Ocular Toxicol. 33, 94-95 (2014).

80. Sherertz, E. F. Acneiform eruption due to "megadose" vitamins B6 and B12. Cutis 48, 119-120 (1991).

81. Dupre, A., Albarel, N., Bonafe, J. L., Christol, B. \& Lassere, J. Vitamin B-12 induced acnes. Cutis 24 210-211 (1979)

82. Braun-Falco, O. \& Lincke, $\mathrm{H}$. The problem of vitamin $\mathrm{B}_{6} / \mathrm{B} 12$ acne. A contribution on acn medicamentosa [German]. MMW Munch. Med. Wochenschr. 118, 155-160 (1976).

83. Puissant, A., Vanbremeersch, F., Monfort, J. \& Lamberton, J. N. A new iatrogenic dermatosis: acne caused by vitamin B 12 [French]. Bull. Soc. Fr. Dermatol. Syphiligr. 74, 813-815 (1967).

84. Johnson, T., Kang, D., Barnard, E \& Li, H. Strain-level differences in porphyrin production and regulation in Propionibacterium acnes elucidate disease associations. mSphere 1, e00023-15 (2016)

85. Palmer, C. N. et al. Common loss-of-function variants of the epidermal barrier protein filaggrin are a major predisposing factor for atopic dermatitis. Nat. Genet. 38, 441-446 (2006)

86. The EArly Genetics and Lifecourse Epidemiology (EAGLE) Eczema Consortium. Multi-ancestry genomewide association study of 21,000 cases and 95,000 controls identifies new risk loci for atopic dermatitis. Nat. Genet. 47, 1449-1456 (2015).

87. Leyden, J. J., Marples, R. R. \& Kligman, A. M. Staphylococcus aureus in the lesions of atopic dermatitis. Br. J. Dermatol. 90, 525-530 (1974).

88. Huang, J. T., Abrams, M., Tlougan, B., Rademaker, A \& Paller, A. S. Treatment of Staphylococcus aureus colonization in atopic dermatitis decreases disease severity. Pediatrics 123, e808-814 (2009).

89. Bath-Hextall, F. J., Birnie, A. J., Ravenscroft, J. C. \& Williams, H. C. Interventions to reduce Staphylococcus aureus in the management of atopic eczema: an updated Cochrane review. Br. J. Dermatol. 163 , 12-26 (2010).

90. Byrd A L et al. Staphylococcus aureus and Staphylococcus epidermidis strain diversity underlying pediatric atopic dermatitis. Sci. Transl Med. 9, eaal4651 (2017)

91. Kennedy, E. A. et al. Skin microbiome before development of atopic dermatitis: early colonization with commensal staphylococci at 2 months is associated with a lower risk of atopic dermatitis at 1 year. J. Allergy Clin. Immunol. 139, 166-172 (2017).

92. Chng, K. R. et al. Whole metagenome profiling reveals skin microbiome-dependent susceptibility to atopic dermatitis flare. Nat. Microbiol. 1, 16106 (2016).

93. Hanski, I. et al. Environmental biodiversity, human microbiota, and allergy are interrelated. Proc. Natl Acad. Sci. USA 109, 8334-8339 (2012).

94. Fyhrquist, N. et al. Acinetobacter species in the skin microbiota protect against allergic sensitization and inflammation. J. Allergy Clin. Immunol. 134 1301-1309.e11 (2014).

95. Zeeuwen, P. L. et al. Gram-positive anaerobe cocci are underrepresented in the microbiome of filaggrindeficient human skin. J. Allergy Clin. Immunol. 139 , 1368-1371 (2017)

96. Nakamura, Y et al Staphylococcus delta-toxin induces allergic skin disease by activating mast cells. Nature 503, 397-401 (2013).

97. Niebuhr, M. et al. Staphylococcal alpha-toxin is a strong inducer of interleukin-17 in humans. Infect. Immun. 79, 1615-1622 (2011).

98. Kaesler, S. et al. Staphylococcus aureus-derived lipoteichoic acid induces temporary T-cell paralysis independent of Toll-like receptor 2. J. Allergy Clin. Immunol. 138, 780-790.e6 (2016).

99. Zhang, L. J. et al. Dermal adipocytes protect against invasive Staphylococcus aureus skin infection. Science 347, 67-71 (2015). 
100. Nakatsuji, T. et al. Staphylococcus aureus exploits epidermal barrier defects in atopic dermatitis to trigger cytokine expression. J. Invest. Dermatol. 136, 2192-2200 (2016)

101. Kobayashi, T. et al. Dysbiosis and Staphylococcus aureus colonization drives inflammation in atopic dermatitis. Immunity 42, 756-766 (2015). This study demonstrates how $S$. aureus predominance can drive skin inflammation in an animal model of altered barrier integrity.

102. Oh, J. et al. The altered landscape of the human skin microbiome in patients with primary immunodeficiencies. Genome Res. 23, 2103-2114 (2013).

103. Smeekens, S. P. et al. Skin microbiome imbalance in patients with STAT $1 /$ STAT3 defects impairs innate host defense responses. J. Innate Immun. 6, 253-262 (2014).

104. Chu, E. Y. et al. Cutaneous manifestations of DOCK8 deficiency syndrome. Arch. Dermatol. 148, 79-84 (2012).

105. Prompers, L. et al. High prevalence of ischaemia, infection and serious comorbidity in patients with diabetic foot disease in Europe. Baseline results from the Eurodiale study. Diabetologia 50, 18-25 (2007).

106. Valensi, P., Girod, I., Baron, F., Moreau-Defarges, T $\&$ Guillon, P. Quality of life and clinical correlates in patients with diabetic foot ulcers. Diabetes Metab. 31 263-271 (2005)

107. Ramsey, S. D. et al. Incidence, outcomes, and cost of foot ulcers in patients with diabetes. Diabetes Care 22 382-387 (1999)

108. Gardner, S. E., Hillis, S. L., Heilmann, K., Segre, J. A $\&$ Grice, E. A. The neuropathic diabetic foot ulcer microbiome is associated with clinical factors. Diabetes 62, 923-930 (2013)

109. Loesche, M. et al. Temporal stability in chronic wound microbiota is associated with poor healing. J. Invest. Dermatol. 137, 237-244 (2017).

110. Martinez, C. et al. Unstable composition of the fecal microbiota in ulcerative colitis during clinical remission. Am. J. Gastroenterol. 103, 643-648 (2008).

111. Kalan, L. et al. Redefining the chronic-wound microbiome: fungal communities are prevalent, dynamic, and associated with delayed healing. $\mathrm{mBio}$ 7, e01058-16 (2016)

112. Korem, T. et al. Growth dynamics of gut microbiota in health and disease inferred from single metagenomic samples. Science 349, 1101-1106 (2015).

113. Segre, J. A. Epidermal barrier formation and recovery in skin disorders. J. Clin. Invest. 116, 1150-1158 (2006)

114. Gallo, R. L. \& Hooper, L. V. Epithelial antimicrobial defence of the skin and intestine. Nat. Rev. Immunol. 12, 503-516 (2012).

115. Kong, H. H. et al. Performing skin microbiome research: a method to the madness. J. Invest. Dermatol. 137, 561-568 (2017).

116. Alexeyev, O. A. Bacterial landscape of human skin: seeing the forest for the trees. Exp. Dermatol. 22, 443-446 (2013).

117. Alexeyev, O. A. \& Jahns, A. C. Sampling and detection of skin Propionibacterium acnes: current status. Anaerobe 18, 479-483 (2012).

118. Grice, E. A. et al. A diversity profile of the human skin microbiota. Genome Res. 18, 1043-1050 (2008).

119. Nakatsuji, T. et al. The microbiome extends to subepidermal compartments of normal skin. Nat. Commun. 4, 1431 (2013).

120. Zeeuwen, P. L. et al. Microbiome dynamics of human epidermis following skin barrier disruption. Genome Biol. 13, R101 (2012).

121. Nagy, I. et al. Propionibacterium acnes and lipopolysaccharide induce the expression of antimicrobial peptides and proinflammatory cytokines/ chemokines in human sebocytes. Microbes Infect. 8, 2195-2205 (2006).

122. Naik, S. et al. Commensal-dendritic-cell interaction specifies a unique protective skin immune signature. Nature 520, 104-108 (2015)

123. Naik, S. et al. Compartmentalized control of skin immunity by resident commensals. Science 337 1115-1119 (2012).

This study shows that skin commensals can promote cutaneous adaptive immunity to pathogens.

124. PrabhuDas, M. et al. Challenges in infant immunity: implications for responses to infection and vaccines. Nat. Immunol. 12, 189-194 (2011).
125. Scharschmidt, T. C. et al. A wave of regulatory $\mathrm{T}$ cells into neonatal skin mediates tolerance to commensal microbes. Immunity 43, 1011-1021 (2015).

This study describes how FoxP3 ${ }^{+} \mathrm{T}$ cells accumulate in neonatal skin and promote tolerance to commensals.

126. Belkaid, Y. \& Tamoutounour, S. The influence of skin microorganisms on cutaneous immunity. Nat. Rev. Immunol. 16, 353-366 (2016). This is a notable Review on the roles of different microorganisms in educating innate and adaptive components of the skin immune system.

127. Belkaid, Y. \& Harrison, O. J. Homeostatic immunity and the microbiota. Immunity 46, 562-576 (2017).

128. Hand, T. W. et al. Acute gastrointestinal infection induces long-lived microbiota-specific T cell responses. Science 337, 1553-1556 (2012).

129. Otto, M. Staphylococcus epidermidis - the 'accidental' pathogen. Nat. Rev. Microbiol. 7 555-567 (2009).

130. Newell, E. W. \& Davis, M. M. Beyond model antigens: high-dimensional methods for the analysis of antigenspecific T cells. Nat. Biotechnol. 32, 149-157 (2014).

\section{Acknowledgements}

This work was supported by the Division of Intramural Research of the National Human Genome Research Institute and the National Institute of Allergy and Infectious Diseases.

\section{Author contributions}

A.L.B., Y.B. and J.A.S. contributed to researching data for article. A.L.B., Y.B. and J.A.S. substantially contributed to the discussion of content. A.L.B and J.A.S. wrote the article. A.L.B., Y.B. and J.A.S. reviewed and edited the manuscript before submission.

Competing interests statement

The authors declare no competing interests.

\section{Publisher's note}

Springer Nature remains neutral with regard to jurisdictional claims in published maps and institutional affiliations. 